\title{
OPERATOR IDEALS AND ASSEMBLY MAPS IN $K$-THEORY
}

\author{
GUILLERMO CORTINANAS AND GISELA TARTAGLIA
}

(Communicated by Brooke Shipley)

\begin{abstract}
Let $\mathcal{B}$ be the ring of bounded operators in a complex, separable Hilbert space. For $p>0$ consider the Schatten ideal $\mathcal{L}^{p}$ consisting of those operators whose sequence of singular values is $p$-summable; put $\mathcal{S}=\bigcup_{p} \mathcal{L}^{p}$. Let $G$ be a group and $\mathcal{V}$ cyc the family of virtually cyclic subgroups. Guoliang $\mathrm{Yu}$ proved that the $K$-theory assembly map

$$
H_{*}^{G}(\mathcal{E}(G, \mathcal{V} c y c), K(\mathcal{S})) \rightarrow K_{*}(\mathcal{S}[G])
$$

is rationally injective. His proof involves the construction of a certain Chern character tailored to work with coefficients $\mathcal{S}$ and the use of some results about algebraic $K$-theory of operator ideals and about controlled topology and coarse geometry. In this paper we give a different proof of Yu's result. Our proof uses the usual Chern character to cyclic homology. Like Yu's, it relies on results on algebraic $K$-theory of operator ideals, but no controlled topology or coarse geometry techniques are used. We formulate the result in terms of homotopy $K$-theory. We prove that the rational assembly map
\end{abstract}

$$
H_{*}^{G}\left(\mathcal{E}(G, \mathcal{F} \text { in }), K H\left(\mathcal{L}^{p}\right)\right) \otimes \mathbb{Q} \rightarrow K H_{*}\left(\mathcal{L}^{p}[G]\right) \otimes \mathbb{Q}
$$

is injective. We show that the latter map is equivalent to the assembly map considered by $\mathrm{Yu}$, and thus obtain his result as a corollary.

\section{INTRODUCTION}

Let $G$ be a group; a family of subgroups of $G$ is a nonempty family $\mathcal{F}$ closed under conjugation and under taking subgroups. If $\mathcal{F}$ is a family of subgroups of $G$, then a $G$-simplicial set $X$ is called a $(G, \mathcal{F})$-complex if the stabilizer of every simplex of $X$ is in $\mathcal{F}$. The category of $G$-simplicial sets can be equipped with a closed model structure where an equivariant map $X \rightarrow Y$ is a weak equivalence (resp. a fibration) if $X^{H} \rightarrow Y^{H}$ is a weak equivalence (resp. a fibration) for every $H \in \mathcal{F}$ (see [4, §1]); the $(G, \mathcal{F})$-complexes are the cofibrant objects in this model structure. By a general construction of Davis and Lück (see [8]) any functor $E$ from the category $\mathbb{Z}$-Cat of small $\mathbb{Z}$-linear categories to the category Spt of spectra which sends category equivalences to equivalences of spectra gives rise to an equivariant homology theory of $G$-spaces $X \mapsto H^{G}(X, E(R))$ for each unital ring $R$, such that if $H \subset G$ is a subgroup, then

$$
H_{*}^{G}(G / H, E(R))=E_{*}(R[H])
$$

Received by the editors March 15, 2012, and, in revised form, April 24, 2012.

2010 Mathematics Subject Classification. Primary 19D50, 19D55, 19 K99.

The first author was partially supported by MTM2007-64704.

Both authors were supported by CONICET and partially supported by grants UBACyT 20020100100386, PIP 112-200801-00900 and MathAmSud project U11Math05. 
is just $E_{*}$ evaluated at the group algebra. The isomorphism conjecture for the quadruple $(G, \mathcal{F}, E, R)$ asserts that if $\mathcal{E}(G, \mathcal{F}) \stackrel{\sim}{\rightarrow} p t$ is a $(G, \mathcal{F})$-cofibrant replacement of the point, then the induced map

$$
H_{*}^{G}(\mathcal{E}(G, \mathcal{F}), E(R)) \rightarrow E_{*}(R[G]),
$$

called an assembly map, is an isomorphism. For the family $\mathcal{F}=\mathcal{A l l}$ of all subgroups, (1.2) is always an isomorphism. The appropriate choice of $\mathcal{F}$ varies with $E$. For $E=K$, the nonconnective algebraic $K$-theory spectrum, one takes $\mathcal{F}=\mathcal{V} c y c$, the family of virtually cyclic subgroups. If $E=K H$ is homotopy $K$-theory, one can equivalently take $\mathcal{F}$ to be either $\mathcal{V}$ cyc or the family $\mathcal{F}$ in of finite subgroups ([2, Thm. 2.4]). If $E$ satisfies certain hypotheses, including excision, one can make sense of the map (1.2) when $R$ is replaced by any, not necessarily unital, ring $A$. These hypotheses are satisfied, for example, when $E=K H$. Under milder hypotheses, which are satisfied for example by $E=K$, (1.2) makes sense for those coefficient rings $A$ which are $E$-excisive, i.e. those for which $E$ satisfies excision (see [4] and Subsection 2.1 below).

The main result of this paper concerns the $K H$-assembly map for $R=\mathcal{L}^{p}$, the Schatten ideal. Recall that $\mathcal{L}^{p}$ is an ideal of the ring $\mathcal{B}$ of bounded operators in a complex separable Hilbert space; it consists of those operators whose sequence of singular values is $p$-summable. Let $\mathcal{S}=\bigcup_{p>0} \mathcal{L}^{p}$; by [5, Thm. 8.2.1] (see also [16, Thm. 4]), $\mathcal{S}$ is $K$-excisive. Thus the assembly map (1.2) with coefficients $K(\mathcal{S})$ makes sense; Guoliang Yu proved in [17 that it is rationally injective. His proof involves the construction of a certain Chern character tailored to work with coefficients $\mathcal{S}$ and the use of some results about algebraic $K$-theory of operator ideals ([5], [16]), and about controlled topology and coarse geometry from [1] and [11.

In this paper we give a different proof of Yu's result. Our proof uses the usual Chern character to cyclic homology. Like Yu's, it relies on results about algebraic $K$-theory of operator ideals from [5] and [16, but no controlled topology or coarse geometry techniques are used. We formulate the result in terms of $K H$; we prove:

Theorem 1.3. Let $p>0$ and $G$ a group. Then the rational assembly map

$$
H_{*}^{G}\left(\mathcal{E}(G, \mathcal{F} \text { in }), K H\left(\mathcal{L}^{p}\right)\right) \otimes \mathbb{Q} \rightarrow K H_{*}\left(\mathcal{L}^{p}[G]\right) \otimes \mathbb{Q}
$$

is injective.

Yu's result follows as a corollary.

Corollary 1.4 ([17, Thm. 1.1]). Let $G$ be any group and let $\mathcal{S}=\bigcup_{p>0} \mathcal{L}^{p}$ be the ring of all Schatten operators. Then the rational assembly map

$$
H_{*}^{G}(\mathcal{E}(G, \mathcal{V} c y c), K(\mathcal{S})) \otimes \mathbb{Q} \rightarrow K_{*}(\mathcal{S}[G]) \otimes \mathbb{Q}
$$

is injective.

The proof of the corollary makes it clear that the two assembly maps are isomorphic, so it really is the same result.

The rest of this paper is organized as follows. In Section 2, after recalling some general facts about equivariant homology spectra, we focus on the case of periodic 
cyclic homology. For example, we show in Proposition 2.2.1 that if $X$ is a $(G, \mathcal{F}$ in $)$ complex and $k \supset \mathbb{Q}$ a field, then

$$
H_{n}^{G}(X, H P(k / k))=\bigoplus_{p \in \mathbb{Z}} H_{n+2 p}^{G}(X, H H(k / k)) .
$$

We use this to show in Proposition 2.2.5 that the assembly map

$$
H_{n}^{G}(\mathcal{E}(G, \mathcal{F} i n), H P(k / k)) \rightarrow H P_{n}(k[G] / k)
$$

is injective for every group $G$. In Section 3 we consider the Connes-Karoubi Chern character

$$
\operatorname{ch}: H^{G}(X, K H(A)) \rightarrow H^{G}(X, H P(A / k))
$$

defined in [4, §8]. We show in Proposition 3.3 that the composite of $c h$ with the operator trace gives an equivalence

$$
c: H^{G}\left(X, K H\left(\mathcal{L}^{1}\right)\right) \otimes \mathbb{C} \stackrel{\sim}{\longrightarrow} H^{G}(X, H P(\mathbb{C} / \mathbb{C}))
$$

for every $(G, \mathcal{F}$ in $)$-complex $X$. From this and the fact that $K H_{*}\left(\mathcal{L}^{1}\right) \cong K H_{*}\left(\mathcal{L}^{p}\right)$ we deduce in Corollary 3.5 that a similar equivalence, which we also call $c$, holds for every $p>0$ :

$$
c: H^{G}\left(X, K H\left(\mathcal{L}^{p}\right)\right) \otimes \mathbb{C} \stackrel{\sim}{\longrightarrow} H^{G}(X, H P(\mathbb{C} / \mathbb{C})) .
$$

Section 4 is concerned with Theorem 1.3, which we prove in Theorem 4.1. The proof uses (1.6) and the injectivity of (1.5). Yu's result, Corollary 1.4, is proved in Corollary 4.2 .

Notation 1.7. By a spectrum we understand a sequence $E=\left\{{ }_{n} E: n \geq 1\right\}$ of simplicial sets and bonding maps $\Sigma\left({ }_{n} E\right) \rightarrow{ }_{n+1} E$; thus our notation differs from that of other authors (e.g. [13]) who use the term prespectrum for such an object. If $E, F: C \rightarrow$ Spt are functorial spectra, then by a (natural) map $f: E \stackrel{\sim}{\longrightarrow} F$ we mean a zig-zag of natural maps

$$
E=Z_{0} \stackrel{f_{1}}{\longrightarrow} Z_{1} \stackrel{f_{2}}{\longleftarrow} Z_{2} \stackrel{f_{3}}{\longrightarrow} \ldots Z_{n}=F
$$

such that each right to left arrow $f_{i}$ is an object-wise weak equivalence. If also the left to right arrows are object-wise weak equivalences, then we say that $f$ is a weak equivalence or simply an equivalence. If $E$ and $F$ are spectra, we write $E \oplus F$ for their wedge or coproduct. The Dold-Kan correspondence associates a spectrum to every chain complex of abelian groups. Although our notation does not distinguish a chain complex from the spectrum associated to it, it will be clear from the context to which of the two we are referring.

Rings in this paper are not assumed unital unless explicitly stated. We use the letters $A, B$ for rings, and $R, S$ for unital rings. If $X$ is a set, then $M_{X}$ is the ring of all matrices $\left(z_{x, y}\right)_{x, y \in X \times X}$ with integer coefficients, only finitely many of which are nonzero. If $A$ is a ring, then $M_{X} A=M_{X} \otimes A$; in particular $M_{X} \mathbb{Z}=M_{X}$. If $A$ and $B$ are rings, then $A \oplus B$ is their direct sum as abelian groups, equipped with coordinate-wise multiplication. 


\section{Equivariant CYCLIC HOMOLOGY}

2.1. Equivariant homology of simplicial sets. Let $k$ be a field. A $k$-linear category is a small category enriched over the category of $k$-vector spaces. We write $k$-Cat for the category of $k$-linear categories and $k$-linear functors. Observe that by regarding a unital $k$-algebra as a $k$-linear category with one object, we obtain a fully faithful embedding of $k$-algebras into $k$-Cat. Let $\mathcal{C} \in k$-Cat and consider the $k$-module

$$
\mathcal{A}(\mathcal{C})=\bigoplus_{x, y \in \mathcal{C}} \operatorname{hom}_{\mathcal{C}}(x, y)
$$

If $f \in \mathcal{A}(\mathcal{C})$ write $f_{a, b}$ for the component in $\operatorname{hom}_{\mathcal{C}}(b, a)$. The multiplication law

$$
(f g)_{a, b}=\sum_{c \in \mathrm{ob} \mathcal{C}} f_{a, c} g_{c, b}
$$

makes $\mathcal{A}(\mathcal{C})$ into an associative $k$-algebra, which is unital if and only if obC is finite. Whatever the cardinal of obC is, $\mathcal{A}(\mathcal{C})$ is always a ring with local units, i.e. a filtering colimit of unital rings. We call $\mathcal{A}(\mathcal{C})$ the arrow ring of $\mathcal{C}$. If $F: \mathcal{C} \mapsto \mathcal{D}$ is a $k$-linear functor which is injective on objects, then it defines a homomorphism $\mathcal{A}(F): \mathcal{A}(\mathcal{C}) \rightarrow \mathcal{A}(\mathcal{D})$ by the rule $\alpha \mapsto F(\alpha)$. Hence we may regard $\mathcal{A}$ as a functor

$$
\mathcal{A}: \text { inj-k-Cat } \rightarrow k \text {-Alg }
$$

from the category of $k$-linear categories and functors which are injective on objects, to the category of $k$-algebras. However $\mathcal{A}(F)$ is not defined for general $k$-linear $F$. Let $E: k$-Cat $\rightarrow$ Spt be a functor. If $R$ is a unital $k$-algebra and $I \triangleleft R$ is a $k$-ideal, we put

$$
E(R: I)=\operatorname{hofiber}(E(R) \rightarrow E(R / I)) .
$$

Thus if we assume $E(0) \stackrel{\sim}{\longrightarrow} *$, we have $E(R: R) \stackrel{\sim}{\longrightarrow} E(R)$. We say that $E$ is finitely additive if the canonical map

$$
E(\mathcal{C}) \oplus E(\mathcal{D}) \rightarrow E(\mathcal{C} \oplus \mathcal{D})
$$

is an equivalence. We assume from now on that $E$ is finitely additive. If $A$ is a not necessarily unital $k$-algebra, write

$$
\tilde{A}_{k}=A \oplus k
$$

for the unitization of $A$ as a $k$-algebra. Put

$$
E(A)=E\left(\tilde{A}_{k}: A\right) .
$$

If $A$ happens to be unital, we have two definitions for $E(A)$; they are equivalent by [3, Lemma 1.1]. A not necessarily unital $\operatorname{ring} A$ is called E-excisive if for any embedding $A \triangleleft R$ as an ideal of a unital $k$-algebra, the canonical map

$$
E(A) \rightarrow E(R: A)
$$

is an equivalence.

Standing Assumptions 2.1.3. We shall henceforth assume that $E: k-\mathrm{Cat} \rightarrow \mathrm{Spt}$ satisfies each of the following:

i) Every algebra with local units is $E$-excisive.

ii) If $H$ is a group and $A$ an $E$-excisive algebra, then $A[H]$ is $E$-excisive.

iii) If $A$ is $E$-excisive, $X$ a set and $x \in X$, then $M_{X} A$ is $E$-excisive, and $E$ sends the map $A \rightarrow M_{X} A, a \mapsto e_{x, x} a$ to a weak equivalence. 
iv) There is a natural weak equivalence $E(\mathcal{A}(\mathcal{C})) \stackrel{\sim}{\longrightarrow} E(\mathcal{C})$ of functors inj $k-$ Cat $\rightarrow$ Spt.

v) Let $A$ and $B$ be algebras, and let $C=A \oplus B$ be their direct sum, with coordinate-wise multiplication. Then $C$ is $E$-excisive if and only if both $A$ and $B$ are. Moreover if these equivalent conditions are satisfied, then the map $E(A) \oplus E(B) \rightarrow E(C)$ is an equivalence.

Examples 2.1.4. The assumptions above can be formulated for linear categories over any commutative, unital ground ring $k$. The (nonconnective) $K$-theory spectrum $K$ satisfies the standing assumptions for $k=\mathbb{Z}$ as well as for any field $k$ of characteristic zero ([4, Prop. 4.3.1, Prop. 6.4]). The homotopy $K$-theory spectrum $K H$ is excisive; i.e. every ring is $K H$-excisive [15]. Furthermore it satisfies the assumptions over any ground ring $k$ ([4, Prop. 5.5]). A $k$-linear category $\mathcal{C}$ has associated a canonical cyclic $k$-module $C(\mathcal{C} / k)([10])$ with

$$
C(\mathcal{C} / k)_{n}=\bigoplus_{\left(c_{0}, \ldots, c_{n}\right) \in \mathrm{obC}^{n+1}} \operatorname{hom}_{\mathcal{C}}\left(c_{1}, c_{0}\right) \otimes_{k} \cdots \otimes_{k} \operatorname{hom}_{\mathcal{C}}\left(c_{0}, c_{n}\right) .
$$

The Hochschild, cyclic, negative cyclic and periodic cyclic homology of $\mathcal{C}$ over $k$ are the respective homologies of $C(\mathcal{C} / k)$; they are denoted $H H(/ k), H C(/ k), H N(/ k)$ and $H P(/ k)$. Both $H H(/ k)$ and $H C(/ k)$ satisfy the assumptions above when $k$ is any field [4, Prop. 6.4]. If $k$ is a field of characteristic zero, then $H P(/ k)$ is excisive [7] furthermore, it satisfies the standing assumptions because $H C(/ k)$ does. It follows that also $H N(/ k)$ satisfies the assumptions.

Let $G$ be a group and $S$ a $G$-set. Write $\mathcal{G}^{G}(S)$ for its transport groupoid. By definition $\operatorname{obG}^{G}(S)=S$, and $\operatorname{hom}_{\mathcal{G}^{G}(S)}(s, t)=\{g \in G: g \cdot s=t\}$. If $R$ is a unital $k$-algebra we consider a small category $R\left[\mathcal{G}^{G}(S)\right]$. The objects of $R\left[\mathcal{G}^{G}(S)\right]$ are those of $\mathcal{G}^{G}(S)$ and

$$
\operatorname{hom}_{R\left[\mathcal{G}^{G}(S)\right]}(s, t)=R \otimes \mathbb{Z}\left[\operatorname{hom}_{\mathcal{G}^{G}(S)}(s, t)\right]
$$

with the obvious composition rule. Note that $R\left[\mathcal{G}^{G}(S)\right]$ is a $k$-linear category. We write $\operatorname{Or} G$ for the orbit category of $G$; its objects are the $G$-sets $G / H, H \subset G$ is a subgroup; its homomorphisms are the $G$-equivariant maps. The rule $G / H \mapsto$ $R\left[\mathcal{G}^{G}(G / H)\right]$ defines a functor $\mathrm{Or} G \rightarrow k$-Cat.

Remark 2.1.5. If $A$ is any not necessarily unital $k$-algebra, we put

$$
\mathcal{A}\left(A\left[\mathcal{G}^{G}(G / H)\right]\right)=\operatorname{ker}\left(\mathcal{A}\left(\tilde{A}_{k}\left[\mathcal{G}^{G}(G / H)\right]\right) \rightarrow \mathcal{A}\left(k\left[\mathcal{G}^{G}(G / H)\right]\right)\right) .
$$

Note that $\mathcal{A}\left(A\left[\mathcal{G}^{G}(G / H)\right]\right)$ is always defined, even though $A\left[\mathcal{G}^{G}(G / H)\right]$ is not. Morover, by [4, Lemma 3.2.6] there is an isomorphism

$$
\mathcal{A}\left(A\left[\mathcal{G}^{G}(G / H)\right]\right) \stackrel{\sim}{\longrightarrow} M_{G / H}(A[H]) .
$$

If $R$ is a unital algebra and $I \triangleleft R$ is a $k$-ideal, put

$$
\begin{aligned}
E(R & {\left.\left[\mathcal{G}^{G}(G / H)\right]: I\left[\mathcal{G}^{G}(G / H)\right]\right) } \\
\quad & =\operatorname{hofiber}\left(E\left(R\left[\mathcal{G}^{G}(G / H)\right]\right) \rightarrow E\left((R / I)\left[\mathcal{G}^{G}(G / H)\right]\right)\right) .
\end{aligned}
$$

In the particular case when $A$ is an $E$-excisive algebra, we set

$$
E\left(A\left[\mathcal{G}^{G}(G / H)\right]\right)=E\left(\tilde{A}_{k}\left[\mathcal{G}^{G}(G / H)\right]: A\left[\mathcal{G}^{G}(G / H)\right]\right) .
$$


If $X$ is a $G$-simplicial set, we consider the coend of the spectra

$$
H^{G}(X, E(R: I))=\int^{\mathrm{Or} G} X_{+}^{H} \wedge E\left(R\left[\mathcal{G}^{G}(G / H)\right]: I\left[\mathcal{G}^{G}(G / H)\right]\right) .
$$

To abbreviate notation, we write $H^{G}(X, E(R))$ for $H^{G}(X, E(R: R))$. The spectrum $H^{G}(X, E(R))$ is a simplicial set version of the Davis-Lück equivariant homology spectrum associated with $E([8,4])$. We have a fibration sequence

$$
H^{G}(X, E(R: I)) \rightarrow H^{G}(X, E(R)) \rightarrow H^{G}(X, E(R / I)) .
$$

If $A$ is $E$-excisive, put

$$
H^{G}(X, E(A)):=H^{G}\left(X, E\left(\tilde{A}_{k}: A\right)\right) .
$$

By [4, Prop. 3.3.9], if

$$
0 \rightarrow A^{\prime} \rightarrow A \rightarrow A^{\prime \prime} \rightarrow 0
$$

is an extension of $E$-excisive algebras, then

$$
E\left(A^{\prime}\left[\mathcal{G}^{G}(-)\right]\right) \rightarrow E\left(A\left[\mathcal{G}^{G}(-)\right]\right) \rightarrow E\left(A^{\prime \prime}\left[\mathcal{G}^{G}(-)\right]\right)
$$

and

$$
H^{G}\left(X, E\left(A^{\prime}\right)\right) \rightarrow H^{G}(X, E(A)) \rightarrow H^{G}\left(X, E\left(A^{\prime \prime}\right)\right)
$$

are homotopy fibrations.

\subsection{Equivariant periodic cyclic homology of $(G, \mathcal{F} i n)$-complexes.}

Proposition 2.2.1. Let $X$ be a $(G, \mathcal{F}$ in $)$-complex, and let $k \supset \mathbb{Q}$ be a field. There is a natural quasi-isomorphism

$$
\bigoplus_{p \in \mathbb{Z}} H^{G}(X, H H(k / k))[2 p] \stackrel{\sim}{\longrightarrow} H^{G}(X, H P(k / k)) .
$$

Proof. It suffices to show that there is a quasi-isomorphism

$$
\bigoplus_{p \in \mathbb{Z}} H^{G}(G / H, H H(k / k))[2 p] \stackrel{\sim}{\longrightarrow} H^{G}(G / H, H P(k / k))
$$

for $H \in \mathcal{F}$ in, natural with respect to $G$-equivariant maps. In particular we may restrict ourselves to proving the proposition for $X$ a discrete, $G$-finite $(G, \mathcal{F}$ in)complex. The cyclic module $C\left(k\left[\mathcal{G}^{G}(X)\right] / k\right)$ decomposes into a direct sum of cyclic modules [4, 7.1]

$$
C\left(k\left[\mathcal{G}^{G}(X)\right] / k\right)=\bigoplus_{[g] \in \operatorname{con} G} C^{[g]}\left(k\left[\mathcal{G}^{G}(X)\right] / k\right) .
$$

Here the direct sum runs over the set $\operatorname{con} G$ of conjugacy classes of elements of $G$. Because $X$ is a $(G, \mathcal{F}$ in $)$-complex, $C^{[g]}\left(k\left[\mathcal{G}^{G}(X)\right] / k\right)=0$ for $g$ of infinite order. So assume $g$ is of finite order. Let $Z_{g} \subset G$ be the centralizer subgroup. By [4. Lemma 7.2] (see also [9, Cor. 9.12]), there is a natural quasi-isomorphism of cyclic $k$-modules

$$
H\left(Z_{g}, k\left[X^{g}\right]\right) \rightarrow C^{[g]}\left(k\left[\mathcal{G}^{G}(X)\right] / k\right) .
$$

Here the domain has the cyclic structure given by

$$
\begin{gathered}
t_{n}: H\left(Z_{g}, k\left[X^{g}\right]\right)_{n}=k\left[Z_{g}\right]^{\otimes n} \otimes_{k} k\left[X^{g}\right] \rightarrow H\left(Z_{g}, k\left[X^{g}\right]\right)_{n}, \\
t_{n}\left(z_{1} \otimes \cdots \otimes z_{n} \otimes x\right)=\left(z_{1} \cdots z_{n}\right)^{-1} g \otimes z_{1} \otimes \cdots \otimes z_{n-1} \otimes z_{n}(x) .
\end{gathered}
$$


Let $\langle g\rangle \subset Z_{g}$ be the cyclic subgroup. A Serre spectral sequence argument (see the proof of [14, Lemma 9.7.6]) shows that the projection

$$
H\left(Z_{g}, k\left[X^{g}\right]\right) \rightarrow H\left(Z_{g} /\langle g\rangle, k\left[X^{g}\right]\right)
$$

is a quasi-isomorphism of cyclic $k$-modules. Summing up we have a natural zig-zag of quasi-isomorphisms of cyclic modules:

$$
\bigoplus_{[g] \in \operatorname{con}_{f} G} H\left(Z_{g} /\langle g\rangle, k\left[X^{g}\right]\right) \stackrel{\sim}{\longleftarrow} \bigoplus_{[g] \in \operatorname{con}_{f} G} H\left(Z_{g}, k\left[X^{g}\right]\right) \stackrel{\sim}{\longrightarrow} C\left(k\left[\mathcal{G}^{G}(X)\right] / k\right) .
$$

Here $\operatorname{con}_{f} G$ is the set of conjugacy classes of elements of finite order. We remark that because $X$ is a finite $(G, \mathcal{F}$ in $)$-complex by assumption, the direct sums above have only finitely many nonzero summands. By [14, Corollary 9.7.2], we have a natural equivalence

$$
H P\left(H\left(Z_{g} /\langle g\rangle, k\left[X^{g}\right]\right)\right) \stackrel{\sim}{\longrightarrow} \prod_{p \in \mathbb{Z}} H\left(Z_{g} /\langle g\rangle, k\left[X^{g}\right]\right)[2 p] .
$$

Summing up we have a natural quasi-isomorphism

$$
\prod_{p \in \mathbb{Z}}\left(\bigoplus_{[g] \in \operatorname{con}_{f} G} H\left(Z_{g}, k\left[X^{g}\right]\right)\right)[2 p] \stackrel{\sim}{\longrightarrow} H P\left(k\left[\mathcal{G}^{G}(X) / k\right]\right) \stackrel{\sim}{\longrightarrow} H^{G}(X, H P(k / k)) .
$$

Taking into account (2.2.2) we obtain a quasi-isomorphism of chain complexes:

$$
\bigoplus_{[g] \in \operatorname{con}_{f} G} H\left(Z_{g}, k\left[X^{g}\right]\right) \stackrel{\sim}{\longrightarrow} H^{G}(X, H H(k / k)) .
$$

Moreover, in (2.2.3) we can replace $\prod_{p \in \mathbb{Z}}$ by $\bigoplus_{p \in \mathbb{Z}}$ because $H_{n}^{G}(X, H H(k / k))=0$ for $n \neq 0$. Indeed, $X$ is a finite disjoint union of homogeneous spaces $G / K$ with $K \in \mathcal{F}$ in, and

$$
\begin{aligned}
H_{n}^{G}(G / K, H H(k / k)) & =H_{n}^{G}(G / K, H H(k / k)) \\
& =H_{n}^{K}(K / K, H H(k / k))=H H_{n}(k[K] / k),
\end{aligned}
$$

which is zero in positive dimensions since $k[K]$ is separable for finite $K$. This concludes the proof.

Proposition 2.2.5 (cf. [9, Rmk. 1.9]). If $k \supset \mathbb{Q}$ is a field, then the assembly map

$$
H_{*}^{G}(\mathcal{E}(G, \mathcal{F} \text { in }), H P(k / k)) \rightarrow H P_{*}(k[G] / k)
$$

is injective.

Proof. The inclusion

$$
C(k[G] / k)=\bigoplus_{[g] \in \operatorname{con} G} C^{[g]}(k[G] / k) \subset \prod_{[g] \in \operatorname{con} G} C^{[g]}(k[G] / k)
$$

induces a chain map $H P(k[G] / k) \rightarrow \prod_{[g] \in \operatorname{con} G} H P^{[g]}(k[G] / k)$. Projecting onto the conjugacy classes of elements of finite order and taking homology, we obtain a homomorphism

$$
H P_{n}(k[G] / k) \rightarrow \prod_{[g] \in \operatorname{con}_{f} G} H P_{n}^{[g]}(k[G] / k)
$$


Now for $g$ of finite order $C^{[g]}(k[G] / k)=H\left(Z_{g}, k\right) \stackrel{\sim}{\longrightarrow} H\left(Z_{g} /\langle g\rangle, k\right)$. Hence by [14. Cor. 9.7.2],

$$
H P_{n}^{[g]}(k[G] / k)=\prod_{m} H_{n+2 m}\left(Z_{g}, k[p t]\right)=\prod_{m} H_{n+2 m}\left(Z_{g}, k\left[\mathcal{E}(G, \mathcal{F} i n)^{g}\right]\right) .
$$

One checks that the composite of the assembly map with the map (2.2.6) is the inclusion

$$
\begin{aligned}
H_{n}^{G}(\mathcal{E}(G, \mathcal{F} i n), H P(k / k))= & \bigoplus_{m} H_{n+2 m}^{G}(\mathcal{E}(G, \mathcal{F} i n), H H(k / k)) \\
& (\text { by Proposition 2.2.1) } \\
\subset & \prod_{m} H_{n+2 m}^{G}(\mathcal{E}(G, \mathcal{F} i n), H H(k / k))=\prod_{m} \bigoplus_{[g] \in \operatorname{con}_{f} G} H_{n+2 m}\left(Z_{g}, k\left[\mathcal{E}(G, \mathcal{F} i n)^{g}\right]\right)
\end{aligned}
$$

(by (2.2.4)

$$
\subset \prod_{m} \prod_{[g] \in \operatorname{con}_{f} G} H_{n+2 m}\left(Z_{g}, k\left[\mathcal{E}(G, \mathcal{F} i n)^{g}\right]\right) .
$$

\section{Equivariant Connes-Karoubi Chern character}

In this section we consider algebras over a field $k$ of characteristic zero. Recall from [4, $\S 8.2$ ] that the homotopy $K$-theory and periodic cyclic homology of a $k$ linear category are related by a Connes-Karoubi Chern character

$$
K H(\mathcal{C}) \stackrel{c h}{\longrightarrow} H P(\mathcal{C} / k) \text {. }
$$

In particular if $G$ is a group, $H \subset G$ a subgroup and $R$ a unital $k$-algebra, we have a map of OrG-spectra,

$$
\operatorname{ch}: K H\left(R\left[\mathcal{G}^{G}(G / H)\right]\right) \rightarrow H P\left(R\left[\mathcal{G}^{G}(G / H)\right] / k\right) .
$$

By [4, Lemma 3.2.6], this map is equivalent to the Chern character

$$
c h: K H(R[H]) \rightarrow H P(R[H] / k)
$$

for each fixed $H$. Using excision, all this extends to an arbitrary nonunital algebra $A$ in place of $R$. We are interested in the particular case when $k$ is either $\mathbb{C}$ or $\mathbb{Q}$ and $A \triangleleft \mathcal{B}$ is an ideal in the algebra $\mathcal{B}$ of bounded operators in a separable complex Hilbert space. Let $p>0$; write $\mathcal{L}^{p} \triangleleft \mathcal{B}$ for the Schatten ideal of those compact operators whose sequence of singular values is $p$-summable. Let $H \subset G$ be a subgroup and $\operatorname{Tr}: \mathcal{L}^{1} \rightarrow \mathbb{C}$ the operator trace. The map of cyclic modules

$$
\begin{aligned}
\operatorname{Tr}: & C\left(\widetilde{\mathcal{L}^{1}}{ }_{\mathbb{C}}\left[\mathcal{G}^{G}(G / H)\right]: \mathcal{L}^{1}\left[\mathcal{G}^{G}(G / H)\right] / \mathbb{C}\right) \rightarrow C\left(\mathbb{C}\left[\mathcal{G}^{G}(G / H)\right] / \mathbb{C}\right), \\
& \operatorname{Tr}\left(a_{0} \otimes g_{0} \otimes \cdots \otimes a_{n} \otimes g_{n}\right)=\operatorname{Tr}\left(a_{0} \cdots a_{n}\right) g_{0} \otimes \cdots \otimes g_{n}
\end{aligned}
$$

induces a natural transformation of $\operatorname{Or} G$-chain complexes:

$$
\operatorname{Tr}: H P\left(\mathcal{L}^{1}\left[\mathcal{G}^{G}(G / H)\right] / \mathbb{C}\right) \rightarrow H P\left(\mathbb{C}\left[\mathcal{G}^{G}(G / H)\right] / \mathbb{C}\right) .
$$

Proposition 3.3. Let $X$ be a $(G, \mathcal{F}$ in $)$-complex and $\mathcal{L}^{1} \triangleleft \mathcal{B}$ the ideal of trace class operators. Then the composite

$$
c: H^{G}\left(X, K H\left(\mathcal{L}^{1}\right)\right) \otimes \mathbb{C} \stackrel{c h}{\longrightarrow} H^{G}\left(X, H P\left(\mathcal{L}^{1} / \mathbb{C}\right)\right) \stackrel{T r}{\longrightarrow} H^{G}(X, H P(\mathbb{C} / \mathbb{C}))
$$

is an equivalence. 
Proof. It suffices to consider the case $X=G / H$ with $H \in \mathcal{F}$ in. By [4, Lemma 3.2.6], we have a homotopy commutative diagram with vertical equivalences:

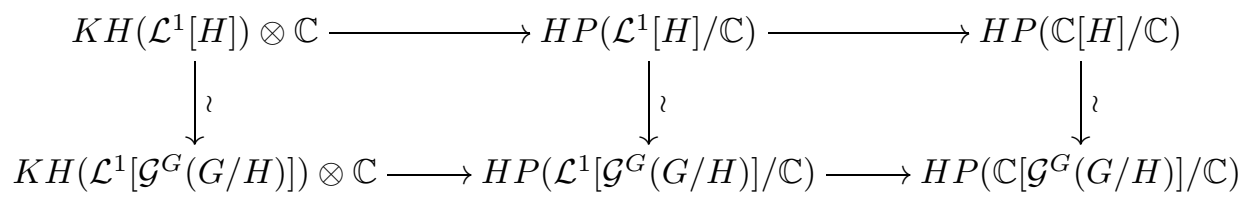

Because $H \in \mathcal{F}$ in, $\mathbb{C}[H]$ is Morita equivalent to its center, which is a sum of copies of $\mathbb{C}$ indexed by the conjugacy classes of $H$ :

$$
\mathbb{C}[H] \sim Z(\mathbb{C}[H])=\bigoplus_{\operatorname{con}(H)} \mathbb{C} .
$$

Since the (periodic) cyclic homology of $\mathbb{C}$ as a $\mathbb{C}$-algebra and as a locally convex topological algebra agree, it follows that the map $H P(\mathbb{C}[H] / \mathbb{C}) \rightarrow H P^{\text {top }}(\mathbb{C}[H])$ is the identity. Next recall from [5, Notation 5.1 and Theorem 6.2.1 (iii)] that if $I \triangleleft \mathcal{B}$ is an operator ideal with a Banach algebra structure such that the inclusion $I \subset \mathcal{B}$ is continuous and $\hat{\otimes}$ is the projective tensor product, then for every locally convex algebra $A$ the comparison map $K H(I \hat{\otimes} A) \rightarrow K^{\mathrm{top}}(I \hat{\otimes} A)$ is an equivalence. In particular this applies when $I=\mathcal{L}^{1}$ and $A=\mathbb{C}[H]$. Hence we have a homotopy commutative diagram with vertical equivalences:

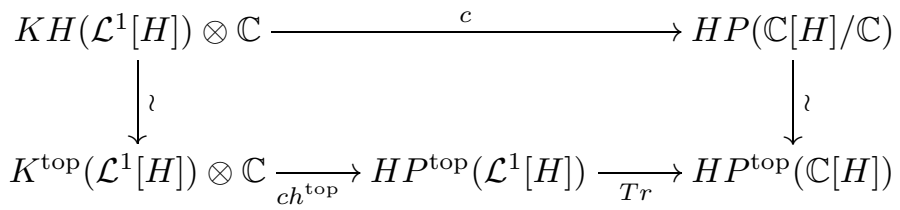

Here $c h^{\text {top }}$ is the topological Connes-Karoubi Chern character. Using (3.4) we have that $T r$ is an equivalence by [6, Prop. 17.2] and $c h^{\text {top }}$ is an equivalence because of (3.4) and the commutativity of the following diagram:

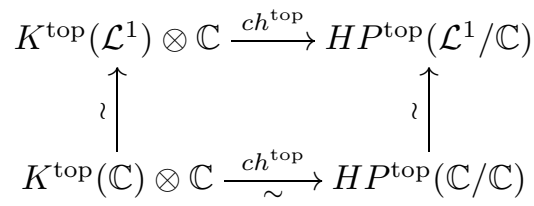

It follows that $c$ is an equivalence. This concludes the proof.

Corollary 3.5. Let $X$ be a $(G, \mathcal{F}$ in $)$ complex. Then, for every $p>0$ there is an equivalence

$$
c: H^{G}\left(X, K H\left(\mathcal{L}^{p}\right)\right) \otimes \mathbb{C} \rightarrow H^{G}(X, H P(\mathbb{C} / \mathbb{C})) .
$$

Proof. Because $\mathcal{L}^{1} / \mathcal{L}^{p}$ for $p<1$ and $\mathcal{L}^{p} / \mathcal{L}^{1}$ for $p>1$ are nilpotent rings and $K H$ is nilinvariant $([15])$, the maps

$$
K H\left(\mathcal{L}^{p}\left[\mathcal{G}^{G}(-)\right]\right) \rightarrow K H\left(\mathcal{L}^{1}\left[\mathcal{G}^{G}(-)\right]\right) \quad(p<1)
$$

and

$$
K H\left(\mathcal{L}^{1}\left[\mathcal{G}^{G}(-)\right]\right) \rightarrow K H\left(\mathcal{L}^{p}\left[\mathcal{G}^{G}(-)\right]\right) \quad(p>1)
$$

are equivalences of $\operatorname{Or} G$-spectra by Remark 2.1.5 and (2.1.8). The proof is now immediate from Proposition 3.3 . 


\section{The $K H$-ASSEMBLy MAP With $\mathcal{L}^{p}$-COEFFICIEntS}

Theorem 4.1. Let $p>0$ and $G$ be a group. Then the rational assembly map

$$
H_{*}^{G}\left(\mathcal{E}(G, \mathcal{F} i n), K H\left(\mathcal{L}^{p}\right)\right) \otimes \mathbb{Q} \rightarrow K H_{*}\left(\mathcal{L}^{p}[G]\right) \otimes \mathbb{Q}
$$

is injective.

Proof. It suffices to show that the map tensored with $\mathbb{C}$ is injective. We have a commutative diagram:

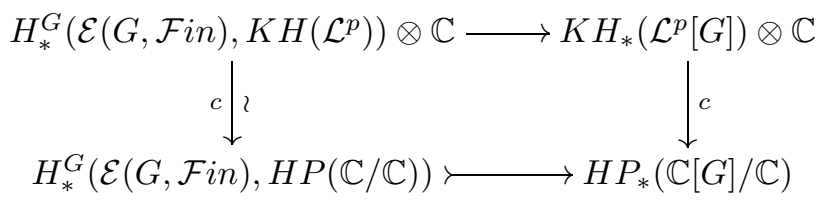

The vertical map on the left is an isomorphism by Corollary 3.5, the bottom horizontal map is injective by Proposition 2.2.5. It follows that the top horizontal map is injective. This concludes the proof.

Let $\mathcal{S}=\bigcup_{p>0} \mathcal{L}^{p}$ be the ring of all Schatten operators. Because $\mathcal{S}^{2}=\mathcal{S}$, the ring $\mathcal{S}$ is $K$-excisive by [12, Thm. C] and [5, Proof of Thm. 8.2.1] (see also [16, Thm. 4]). We can now deduce the following result of Guoliang Yu.

Corollary 4.2 ([17, Thm. 1.1]). The rational assembly map

$$
H_{*}^{G}(\mathcal{E}(G, \mathcal{V} c y c), K(\mathcal{S})) \otimes \mathbb{Q} \rightarrow K_{*}(\mathcal{S}[G]) \otimes \mathbb{Q}
$$

is injective.

Proof. Because $\mathcal{S}=\mathcal{S}^{2}$ and the tensor product of operators $\mathcal{B} \otimes \mathcal{B} \rightarrow \mathcal{B}(H \otimes H) \cong \mathcal{B}$ sends $\mathcal{L}^{1} \otimes \mathcal{S} \rightarrow \mathcal{S}$, the operator ideal $\mathcal{S}$ is sub-harmonic in the sense of [5, Def. 6.5.1]. Hence the map $K\left(A \otimes_{\mathbb{C}} \mathcal{S}\right) \rightarrow K H\left(A \otimes_{\mathbb{C}} \mathcal{S}\right)$ is an equivalence for every H-unital $\mathbb{C}$-algebra $A$ ([5, Thm. 8.2.5, Rmk. 8.2.6]). Applying this when $A=\mathbb{C}[H]$ and using the fact that both $K$ and $K H$ satisfy Standing Assumptions 2.1.3, we obtain an equivalence of $\operatorname{Or} G$-spectra $K\left(\mathcal{S}\left[\mathcal{G}^{G}(G / H)\right]\right) \rightarrow K H\left(\mathcal{S}\left[\mathcal{G}^{G}(G / H)\right]\right.$ ) (see Remark 2.1.5). Hence for every $G$-simplicial set $X$ we have a homotopy commutative diagram with vertical equivalences:

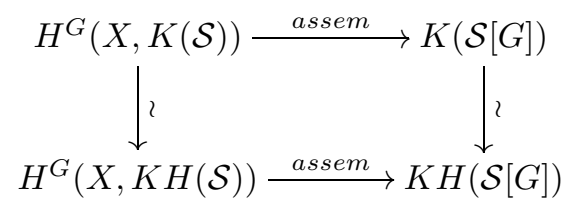

On the other hand, by [2, Thm. 7.4] and (2.1.9), for every ring $A$ the map

$$
\mathcal{E}(G, \mathcal{F} \text { in }) \rightarrow \mathcal{E}(G, \mathcal{V} c y c)
$$

induces a weak equivalence

$$
H^{G}(\mathcal{E}(G, \mathcal{F} \text { in }), K H(A)) \stackrel{\sim}{\longrightarrow} H^{G}(\mathcal{E}(G, \mathcal{V} \text { cyc }), K H(A)) .
$$

Applying this to $A=\mathcal{S}$ and using Theorem 4.1 we obtain that when $X=\mathcal{E}(G, \mathcal{V} c y c)$ the bottom horizontal arrow in (4.3), and thus also the top arrow, is a rational equivalence. 


\section{REFERENCES}

[1] Arthur Bartels, Tom Farrell, Lowell Jones, and Holger Reich, On the isomorphism conjecture in algebraic K-theory, Topology 43 (2004), no. 1, 157-213, DOI 10.1016/S00409383(03)00032-6. MR2030590 (2004m:19004)

[2] Arthur Bartels and Wolfgang Lück, Isomorphism conjecture for homotopy K-theory and groups acting on trees, J. Pure Appl. Algebra 205 (2006), no. 3, 660-696, DOI 10.1016/j.jpaa.2005.07.020. MR2210223(2007e:19005)

[3] Guillermo Cortiñas, The obstruction to excision in K-theory and in cyclic homology, Invent. Math. 164 (2006), no. 1, 143-173, DOI 10.1007/s00222-005-0473-9. MR2207785 (2006k:19006)

[4] G. Cortiñas and E. Ellis, Isomorphism conjectures with proper coefficients. arXiv:1108.5196

[5] Guillermo Cortiñas and Andreas Thom, Comparison between algebraic and topological K-theory of locally convex algebras, Adv. Math. 218 (2008), no. 1, 266-307, DOI 10.1016/j.aim.2007.12.007. MR2409415 (2009h:46136)

[6] Joachim Cuntz, Cyclic theory and the bivariant Chern-Connes character, Noncommutative geometry, Lecture Notes in Math., vol. 1831, Springer, Berlin, 2004, pp. 73-135, DOI 10.1007/978-3-540-39702-1_2. MR2058473(2005e:58007)

[7] Joachim Cuntz and Daniel Quillen, Excision in bivariant periodic cyclic cohomology, Invent. Math. 127 (1997), no. 1, 67-98, DOI 10.1007/s002220050115. MR1423026 (98g:19003)

[8] James F. Davis and Wolfgang Lück, Spaces over a category and assembly maps in isomorphism conjectures in $K$ - and L-theory, K-Theory 15 (1998), no. 3, 201-252, DOI 10.1023/A:1007784106877. MR:1659969(99m:55004)

[9] Wolfgang Lück and Holger Reich, Detecting K-theory by cyclic homology, Proc. London Math. Soc. (3) 93 (2006), no. 3, 593-634, DOI 10.1017/S0024611506015954. MR2266961 (2007h:19008)

[10] Randy McCarthy, The cyclic homology of an exact category, J. Pure Appl. Algebra 93 (1994), no. 3, 251-296, DOI 10.1016/0022-4049(94)90091-4. MR1275967 (95b:19002)

[11] John Roe, Coarse cohomology and index theory on complete Riemannian manifolds, Mem. Amer. Math. Soc. 104 (1993), no. 497, x+90. MR.1147350 (94a:58193)

[12] Andrei A. Suslin and Mariusz Wodzicki, Excision in algebraic K-theory, Ann. of Math. (2) 136 (1992), no. 1, 51-122, DOI 10.2307/2946546. MR1173926 (93i:19006)

[13] R. W. Thomason, Algebraic K-theory and étale cohomology, Ann. Sci. École Norm. Sup. (4) 18 (1985), no. 3, 437-552. MR826102 (87k:14016)

[14] Charles A. Weibel, An introduction to homological algebra, Cambridge Studies in Advanced Mathematics, vol. 38, Cambridge University Press, Cambridge, 1994. MR1269324 (95f:18001)

[15] Charles A. Weibel, Homotopy algebraic K-theory, Algebraic K-theory and algebraic number theory (Honolulu, HI, 1987), Contemp. Math., vol. 83, Amer. Math. Soc., Providence, RI, 1989, pp. 461-488, DOI 10.1090/conm/083/991991. MR991991(90d:18006)

[16] Mariusz Wodzicki, Algebraic K-theory and functional analysis, First European Congress of Mathematics, Vol. II (Paris, 1992), Progr. Math., vol. 120, Birkhäuser, Basel, 1994, pp. 485496. MR 1341858 (97f:46112)

[17] G. Yu, The algebraic K-theory Novikov conjecture for group algebras over the ring of Schatten class operators. arXiv:1106.3796

Departamento de Matemática-IMAS, FCEyN-UBA, Ciudad Universitaria, 1428 Buenos

Aires, Argentina

E-mail address: gcorti@dm.uba.ar

URL: http://mate.dm.uba.ar/〜gcorti

Departamento de Matemática-Imas, FCEyN-UBA, Ciudad Universitaria, 1428 Buenos Aires, Argentina

E-mail address: gtartag@dm.uba.ar 